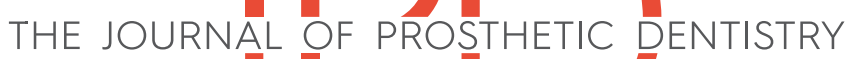

\section{RESEARCH AND EDUCATION \\ Effect of polishing procedures and hydrothermal aging on wear characteristics and phase transformation of zirconium dioxide}

\author{
Darrell Bartolo, BSc, ${ }^{a}$ Glenn Cassar, B. Eng, PhD, ${ }^{b}$ Nadin Al-Haj Husain, MSc, ${ }^{c}$ Mutlu Özcan, DDS, DMD, PhD, \\ and Josette Camilleri, BChD, MPhil, PhD, FIMMM, FADM ${ }^{e}$
}

Zirconia is an oxide ceramic consisting of a pure crystalline matrix having 3 allotropes: monoclinic, tetragonal, and cubic. $^{1-3}$ Monoclinic zirconia exists below a temperature of $1170^{\circ} \mathrm{C}$ and is the weakest of the 3 allotropes. Tetragonal zirconia occurs within the range of $1170^{\circ} \mathrm{C}$ to $2370^{\circ} \mathrm{C}$, while cubic zirconia is found at temperatures higher than $2370^{\circ} \mathrm{C}$.

The physical and mechanical properties of tetragonal zirconia are important when it is used as a dental material. This phase is stabilized at room temperature by the addition of compounds such as yttria $\left(\mathrm{Y}_{2} \mathrm{O}_{3}\right)$ or magnesia $(\mathrm{MgO})$ to form partially stabilized zirconia. This mixture of zirconia in different phases has

\begin{abstract}
Statement of problem. Yttria-stabilized zirconia used for the fabrication of crowns and fixed prostheses may require intraoral adjustments after placement and cementation. Grinding and polishing methods may result in changes in the surface characteristics of zirconia.

Purpose. The purpose of this in vitro study was to assess the effect of polishing procedures on surface roughness, topographical and phase changes of zirconia, and wear of the opposing dentition.

Material and methods. Presintered and precut yttria-stabilized zirconia specimens $(10 \times 10 \times 1 \mathrm{~mm})$ were divided into 4 groups (control, Intensiv, Shofu, 3M ESPE) depending on the polishing method used to prepare the specimens. All tests were carried out in triplicate. The specimens were polished depending on the polishing regimen, while the control was left untreated. The specimens were thermocycled for 3000 cycles, with a temperature range of $5^{\circ} \mathrm{C}$ to $55^{\circ} \mathrm{C}$. The surface roughness, elemental, and phase changes caused by polishing before and after thermocycling were assessed with surface profilometry, energy-dispersive spectroscopy, and X-ray diffraction analysis. The wear on antagonist steatite balls was also measured after mastication simulation. Statistical analysis was performed using 1-way ANOVA and the Tukey post hoc test to perform multiple comparison tests $(\alpha=.05)$.

Results. The polishing procedures increased surface roughness $(\mathrm{Ra})$ of yttria-stabilized zirconia from 0.52 for the control specimen to 0.73 for Intensiv, 0.70 for Shofu, and 0.70 for $3 \mathrm{M} \mathrm{ESPE}(P<.05)$, which was reduced by thermocycling to 0.44 (control), 0.58 (Intensiv), and 0.58 (Shofu) $(P<.001)$, while roughness remained unchanged for $3 \mathrm{M}$ ESPE specimens $(0.75 ; P=.452)$. The deposition of aluminum when using Shofu abrasives and nickel in Intensiv was demonstrated. Phase changes were observed on the zirconia surface with formation of the monoclinic phase in all polishing methods. Specimen aging enhanced the surface phase changes and also induced compressive stresses in zirconia polished with Intensiv. The different polishing protocols did not affect the wear to the antagonist $(P>.05)$.
\end{abstract}

Conclusions. Polishing zirconia increased surface roughness and led to surface phase changes, but wear to the antagonist was not affected. (J Prosthet Dent 2016;

Support provided for the financing of the testing by European Regional Development Fund (Malta) through the project "Developing an Interdisciplinary Material Testing and Rapid Prototyping

R\&D Facility" (Ref. 012).

${ }^{a}$ Graduate student, Department of Restorative Dentistry, Faculty of Dental Surgery, University of Malta, Malta.

becturer, Head of Department, Department of Metallurgy and Materials Engineering, Faculty of Engineering, University of Malta, Malta.

'Graduate student, University of Zurich, Dental Materials Unit, Center for Dental and Oral Medicine, Clinic for Fixed and Removable Prosthodontics and Dental Materials Science, Zurich, Switzerland.

${ }^{d}$ Professor, University of Zurich, Dental Materials Unit, Center for Dental and Oral Medicine, Clinic for Fixed and Removable Prosthodontics and Dental Materials Science, Zurich, Switzerland.

${ }^{\mathrm{e}}$ Associate Professor, Department of Restorative Dentistry, Faculty of Dental Surgery, University of Malta, Malta. 


\section{Clinical Implications}

The use of high-speed polishing rotary instruments is contraindicated for clinical use because of nickel contamination, phase changes in zirconia, and the resultant surface roughness.

greatly improved mechanical properties. Zirconia has high flexural strength and fracture toughness compared with other ceramics and can be used to replace metal frameworks in crowns and fixed dental prostheses. ${ }^{1-3}$

Ahead of a crack tip, zirconia can undergo transformation toughening by transforming the tetragonal zirconia into monoclinic zirconia. Monoclinic zirconia is less dense than tetragonal zirconia because of a resultant expansion of approximately $4 \%$. This produces a compressive stress, which counteracts the external tensile forces and acts to interrupt crack initiation and the propagation of preexisting cracks. Physical and chemical changes have been shown to occur in zirconia during processing procedures.

Various factors have been shown to affect the flexural strength of zirconia. These include speed of polishing, use of airborne-particle abrasion, type of surface preparation, and polishing procedures. Continuous high-speed grinding, as opposed to periodic grinding, with a highspeed handpiece can affect the flexural strength of zirconia. ${ }^{4}$ Similarly, airborne-particle abrasion increases the zirconia flexural strength, ${ }^{5}$ as this activates the phase transformation change from tetragonal to monoclinic, ${ }^{5-8}$ while surface treatments such as argon ion bombardment, and gas plasma results cause limited damage.

Mechanical surface polishing is also affected by the type of particle used, with zirconia causing less damage than alumina. ${ }^{9}$ Damage or roughening caused by polishing can also be reduced by using finer particles, but this weakens the material's surface by increasing microfractures and decreasing fracture toughness. However, coarse polishing has the opposite effect. ${ }^{10}$ Finishing procedures such as glazing reduce flexural strength, ${ }^{11}$ and additional firing for veneering porcelain does not accelerate low-temperature degradation. ${ }^{12}$ Overall, most of the zirconia polishing systems available show clinically acceptable results, with limited surface roughness and no phase transformation occurring during polishing procedures. ${ }^{13}$

Aging also reduces the strength of zirconia significantly with monolithic phase formation. ${ }^{14}$ The resistance to fracture and the phase stability are susceptible to hydrothermal and mechanical conditions. ${ }^{14}$ Thus, the final surface roughness of the zirconia is important as it affects antagonist wear. High-luster polishing has been preferred over glazing of zirconia because it produces less surface roughness and less antagonist wear. ${ }^{15-19}$
Table 1. Type of polishing methods used to polish zirconia surfaces

\begin{tabular}{llll}
\hline Material & Manufacturer & Chemical Composition & \multicolumn{1}{c}{$\begin{array}{c}\text { Clinical } \\
\text { Procedure }\end{array}$} \\
\hline DB FG 9205 & Intensiv & $\begin{array}{l}\text { Diamond particles } \\
\text { imbedded into binder } \\
\text { mix matrix }\end{array}$ & Polishing \\
\hline $\begin{array}{l}\text { 6BG FG 0413, } \\
\text { FG 0414, }\end{array}$ & Shofu & Silicon carbide polishers & $\begin{array}{l}\text { Prepolish, polish, } \\
\text { and superpolish }\end{array}$ \\
\hline FG 0415 & SM ESPE & $\begin{array}{l}\text { Urethane coated paper } \\
\text { with Al oxide grits }\end{array}$ & Finishing, polishing \\
\hline
\end{tabular}

The objectives of this study were to assess the in situ effect of polishing procedures on surface roughness, the topographical and phase changes of yttria-stabilized zirconia, and the wear of the opposing dentition. The null hypotheses were that polishing systems would not show a significant difference in the surface roughness, in the phase change of zirconia, or on the wear of opposing natural teeth.

\section{MATERIAL AND METHODS}

Specimens were cut from zirconia $\left(\mathrm{ZrO}_{2}, \mathrm{Y}_{2} \mathrm{O}_{3}\right)$ blocks (Katana Zirconia HT; Kuraray Noritake) with a grain size of $<0.5 \mu \mathrm{m}$, flexural strength of $>900 \mathrm{MPa}$, and fracture toughness of $5 \mathrm{MPa} \cdot \mathrm{m}^{1 / 2}$. A water-cooled electrical precision diamond wire saw (Well; Walter Ebner) with blade diameter of $0.17 \mathrm{~mm}$ and $30 \mu \mathrm{m}$ roughness was used to cut the specimens. The surfaces of the sectioned specimens were polished manually with P2400 grit silicon carbide paper (Struers) under water cooling until a flat surface was obtained and the thickness was verified with a digital micrometer (Mitutoyo). The specimens were then sintered in a high-temperature furnace (LHT02L16; Nabertherm) at $1500^{\circ} \mathrm{C}$ for 7 hours according to the manufacturer's instructions. Finally, specimens of $1200 \times 1200 \times 260 \mu \mathrm{m} \pm 20 \mu \mathrm{m}$ were obtained after sintering $(10 \times 10 \mathrm{~mm})$. Three grinding and polishing methods were investigated. The types of grinding methods used are shown in Table 1.

The zirconia specimens were individually clamped on a fixed device to avoid movement during preparation. Three out of 4 equally divided portions of the specimens were polished with the same procedure to ensure repeatability of the readings from the same specimen, leaving 1 section unprepared to be used as control. Each specimen was then polished systematically for the corresponding duration (Table 2). The time allowed for each finishing procedure was collectively 60 seconds for the use of multiple types of rotary instruments. A high-speed handpiece with water spray coolant (Sirona) was used for $\mathrm{SH}$ (Shofu) and IN (Intensiv) polishing rotary instruments, while the slow-speed handpiece (Sirona) was used for the M (3M ESPE) polishing rotary instruments. After preparation, the specimens were characterized, thermocycled, and remeasured. 
Table 2. Duration for each polishing regimen

\begin{tabular}{llcc}
\hline Bur & Type of Instrument & Time (sec) & Total Time (sec) \\
\hline Intensiv (IN) & FG 9205 & 60 & 60 \\
\hline Shofu (SH) & 6Brownie (FG 0413) & 20 & 60 \\
\hline & Greenie (FG 0414) & 20 & \\
\hline & Super-greenie (FG 0415) & 20 & \\
\hline 3M ESPE (M) & Course & 15 & 60 \\
\hline & Medium & 15 & \\
\hline & Fine & 15 & \\
\hline & Super fine & 15 \\
\hline
\end{tabular}

The surface roughness, phase, and microstructural changes induced by specimen grinding were investigated before and after thermocycling for 3000 cycles (THE 1100; SD Mechatronik) in distilled water at an alternating temperature of between $5^{\circ} \mathrm{C}$ and $55^{\circ} \mathrm{C}$. The specimens were characterized by energy-dispersive spectroscopy (EDS) and x-ray diffraction analyses (XRD).

Surface analysis of zirconia surfaces was carried out using noncontact 3-dimensional (3D) optical profilometer (Xyris 2000WL; TaiCaan Technologies) based on a precision motion system and a confocal polychromatic (white) light optical probe to measure the displacement at the material surface over a specified area. The region measured $(2 \times 2 \mathrm{~mm})$ was at the center of each specimen, where measurements were made for 201 points on the $\mathrm{x}$-axis and 201 points on the $\mathrm{y}$-axis. The data were subsequently analyzed for the changes in real roughness using software (BODDIES) of the profilometer. BODDIES is a software suite for the analysis and visualization of surface topography data. Software tools are included for the extraction of quantitative textural and geometric characterization of both profile (2-dimensional) and areal (3D) parameters. Average surface roughness (Ra) and mean root square roughness $(\mathrm{Rq})$ were measured in triplicate. The measurement of these parameters enabled the comparison of the treated specimens with the control and with each other. EDS analysis of thermocycled and nonthermocycled specimens was carried out to assess the elemental composition of the materials. The zirconia specimens were mounted on aluminum stubs, coated with carbon, and viewed with a scanning electron microscope (Zeiss MERLIN Field Emission SEM; Carl Zeiss NTS GmbH).

XRD was used to analyze the crystalline structure of the zirconia specimens. X-ray diffraction analysis was performed both on the zirconia surface in grazing incidence asymmetric Bragg (GIAB) mode and also in Bragg Brentano geometry. The diffractometer (D8 Advance; Bruker) used $\mathrm{CuK} \alpha$ radiation at $40 \mathrm{~mA}$ and $45 \mathrm{kV}$, and the detector was rotated between 20 and 70 degrees with a step of 0.02 degrees $2 \theta$ and a step time of 0.5 seconds. The specimens were continuously rotated at 15 revolutions per minute. Near-surface analysis was performed using a Rigaku Ultima IV with a $\mathrm{CuK} \alpha$ source set in grazing incidence mode with an incidence angle of 3 degrees. The diffractometer was operated at $40 \mathrm{~mA}$ and $45 \mathrm{kV}$ from 20 to 70 degrees $2 \theta$ range with a sampling width of 0.05 degrees, and a scan speed 0.5 degrees/ minute. The specimens were again continuously rotated at 15 revolutions per minute. Phase identification was accomplished using a search match software indexing the peaks against Power Diffraction Files (PDF) data provided by the International Centre for Diffraction Data (ICDD).

A mastication simulator (SD Mechatronik $\mathrm{GmbH}$ ) was used to assess the degree of wear to the antagonist in contact with a zirconia surface. For wear testing, antagonists consisted of steatite ball $6 \mathrm{~mm}$ in diameter, which effectively represented the enamel cusps of the teeth. Steatite balls have been used to replace tooth antagonists in a previous study. ${ }^{20}$ The antagonists as well as the zirconia specimens were embedded in special holders with autopolymerizing polymethyl methacrylate (PMMA). A layer of paraffin wax was used to cover the underside of the steatite ball to avoid adhesion to the PMMA. The parameters chosen for the mastication simulator were a loading force of $50 \mathrm{~N}$ with 250000 cycles, vertical speed of $30 \mathrm{~mm} / \mathrm{second}$, and a lateral movement of $0.7 \mathrm{~mm}$ from forward to backward. ${ }^{21-23}$ The number of cycles was sufficient to cause changes in the antagonist. ${ }^{21}$ An average of between 240000 and 250000 mastication simulator cycles conforms with 1 year of in vivo wear. ${ }^{22}$ The difference between using no lateral movements and $0.7 \mathrm{~mm}$ lateral movements led to 8 times more wear. ${ }^{23}$ A force of $50 \mathrm{~N}$ was used as this is the average force found between the antagonists orally. ${ }^{23}$ High-impact velocity $(60 \mathrm{~mm} / \mathrm{second})$ would produce a bouncing effect in a single mastication cycle because of the stiffness of the specimen. ${ }^{24,25}$ Therefore, half that impact velocity was used to avoid the bouncing effect. The testing was performed under water for approximately 36 hours at a frequency of $1.8 \mathrm{~Hz}$. The specimens were thermocycled for 3000 cycles before testing.

The wear on the steatite balls was assessed by profilometric analysis. The 3D image created by the profilometer scan was leveled across the wear surface. From this graph, the width of the worn area was calculated with the following formula:

$\mathrm{VCAP}=1 / 3 \pi \mathrm{h} 2(3 \mathrm{R}-\mathrm{h})$,

where VCAP is the volume of a sphere, $\mathrm{h}$ is the wear height calculated by the Pythagoras theorem from the width of worn area, and $\mathrm{R}$ is the radius of the sphere. The steatite ball was assumed to be a perfect sphere with a radius of $3 \mathrm{~mm}$, and on this basis, the percentage of residual height and volume of the steatite ball after mastication simulation was calculated. 


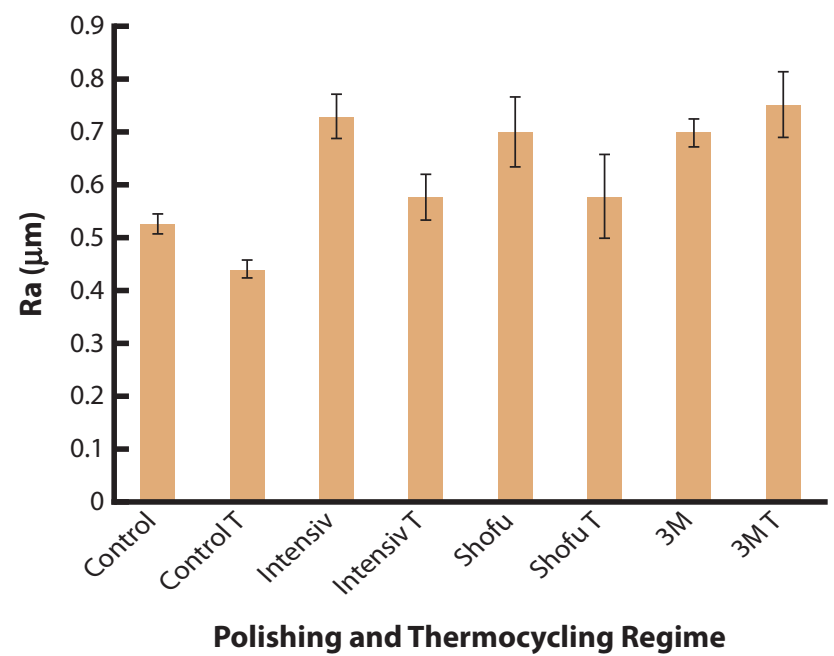

Figure 1. Surface roughness before and after thermocycling (T) of different abrasives on zirconia surface $(n=3)$.

The data were evaluated using statistical software (PASW Statistics v18; SPSS Inc). Parametric tests performed as Kolmogorov-Smirnov tests on the results indicated that the data were normally distributed. ANOVA $(\alpha=.05)$ and the Tukey-Kramer post hoc test $(\alpha=.05)$ were used to perform multiple comparison tests.

\section{RESULTS}

The polishing procedures increased the surface roughness (Ra) of yttria-stabilized zirconia (Fig. 1) from 0.52 $\mu \mathrm{m}$ for the control specimen to $0.73 \mu \mathrm{m}$ for Intensiv, 0.70 for Shofu, and $0.70 \mu \mathrm{m}$ for $3 \mathrm{M}$ ESPE $(P<.05)$, which was reduced by thermocycling to $0.44 \mu \mathrm{m}$ (control), $0.58 \mu \mathrm{m}$ (Intensiv), and $0.58 \mu \mathrm{m}$ (Shofu) $(P<.001)$, while roughness remained unchanged for the $3 \mathrm{M}$ ESPE specimens $(0.75 \mu \mathrm{m} ; P=.452)$.

Zirconia exhibited peaks for zirconium, yttrium, oxygen, and hafnium according to the EDS spectra (Fig. 2). The elemental composition did not vary with or without thermocycling for all specimens except those polished with $\mathrm{SH}$ abrasives. SH polished nonthermocycled specimens showed traces of aluminum, but they were eliminated after thermocyling.

The XRD analysis of the nonthermocyled and thermocycled specimens (Fig. 3A) showed peaks (111), (200), (220), (311), (222) for yttrium zirconium oxide (ICDD: 04-016-2103) in the tetragonal phase. Thermocycling led to a peak shift to the left of the ordinate in the zirconia polished with IN (Fig. 3A, inset). The surface XRD analysis of the nonthermocycled control specimens showed peaks of tetragonal (ICDD: 04-06-2103) yttrium zirconium oxide (111), (200), (220), (311), (222) (Fig. 3B). All the polished specimens exhibited the same peaks except for IN which demonstrated additional peaks for monoclinic (ICDD: 04-005-4476) yttria-stabilized zirconia (111) and Ni (111) and Ni (200) (ICDD: 04-013-4763). However, all thermocycled specimens exhibited peaks of monoclinic yttrium zirconium oxide peak (111) together with the tetragonal peaks. The peaks Ni (111) and Ni (200) of the IN polished specimens were present before and after thermocycling.

The zirconia specimens did not show signs of wear at the end of the 250000 cycles. However, steatite balls exhibited observable wear. The zirconia wore the steatite considerably, with a difference in height between the steatite and steatite after contact with the specimens polished using the different regimens ( $P=.001$; control, $P<.001$; Intensiv, $P=.005$; Shofu, and $P<.001$; 3M ESPE) (Fig. 4). However, no significant difference was noted between steatite balls in contact with unpolished and polished zirconia $(P>.05)$. Also, no significant difference in residual wear volume was noted $(P>.05)$.

\section{DISCUSSION}

In vitro research simulating clinical situations is essential in order to predict material performance in vivo and also the effect it has on the opposing dentition. In this study, zirconia was subject to a simulated process of polishing procedures usually undertaken after insertion when adjustments are necessary. The zirconia would have already been sintered and polished in the laboratory, and the polishing procedures would only serve for polishing the zirconia structure after the modifications undertaken intraorally. The changes are usually performed occlusally to remove any premature contacts and produce a balanced occlusion. ${ }^{7,8,15}$

Polishing procedures performed in anatomic contour zirconia are of more importance because a highly polished, glossy finish with minimal surface roughness could prevent antagonistic tooth wear during mastication. High-luster polishing has been preferred over glazing of zirconia because it produces less surface roughness and less antagonist wear. ${ }^{16-19}$ In the current study, 3 polishing protocols with finishing rotary instruments and disks were investigated. The surface roughness created by the different polishing methods was assessed using a noncontact optical profilometer. The white light confocal laser together with the use of a noncontact version enabled precise assessment of the wear and surface roughness of both the zirconia and the steatite used to simulate the antagonist tooth. Polishing zirconia specimens increased their surface roughness significantly compared with the control.

Thermocycling enabled the test specimens to encounter other variables normally present in in vivo situations. The temperature fluctuations and exposure to water affected the surface roughness. The surface roughness of the IN and $\mathrm{SH}$ polished zirconia specimens decreased after thermocycling, in agreement with a previous study that demonstrated that different aging regimens affected the properties of zirconia. ${ }^{14}$ 

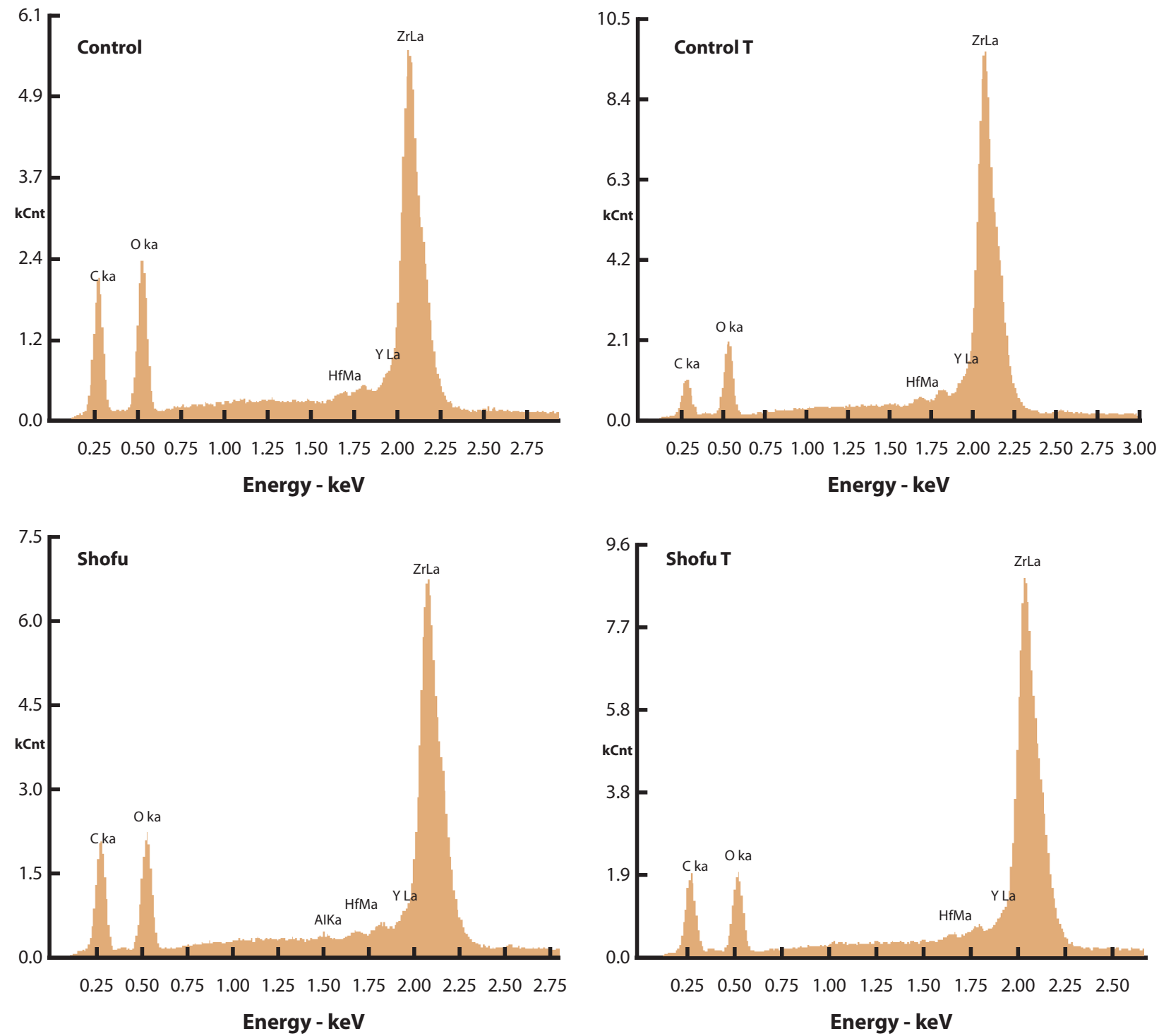

Figure 2. Energy-dispersive spectroscopic analysis of zirconia control and after polishing with Shofu abrasives before and after thermocycling ( $\mathrm{T}$ ).

Phase changes in zirconia that occur during polishing procedures may in turn affect the durability of the material during use. Phase changes in zirconia were assessed by XRD using the Bragg-Brentano methodology. Furthermore, because any changes to the zirconia surface may be limited to the very near surface of the materials, grazing incidence diffraction at a fixed angle of 3 degrees was also used to investigate. Using the grazing angle, XRD crystalline structures on the material surface can be assessed because the $\mathrm{x}$-ray beam is directed at a specific angle, as opposed to powder diffractometry, where the bulk of the material is investigated. The zirconia used in the current study was tetragonal and was not affected by the polishing methods. Thermocycling of zirconia after polishing with Intensiv polishers resulted in a peak shift to lower $2 \theta$ angles, and thus to the left of the $x$-axis. The peak shift was minimal, indicating stresses in the compressive mode leading to an expansion of the crystal lattice. The surface analyses demonstrated monoclinic peaks of zirconia along with the tetragonal peaks. Thus, zirconia polishing resulted in phase changes on the material surface.

Peaks of nickel were found on the Intensiv polished zirconia before and after thermocycling, which can be ascribed to the Ni binder matrix present in the diamond polishing bur that has contaminated the zirconia specimen. The outer surface analysis of the Intensiv polished zirconia also resulted in monoclinic peak (111) being detected both before and after thermocycling, while the other specimens exhibited this peak only after thermocycling. This observation suggests that polishing with Intensiv induces phase changes with more ease than other treated specimens and that aging enhances the phase changes. 

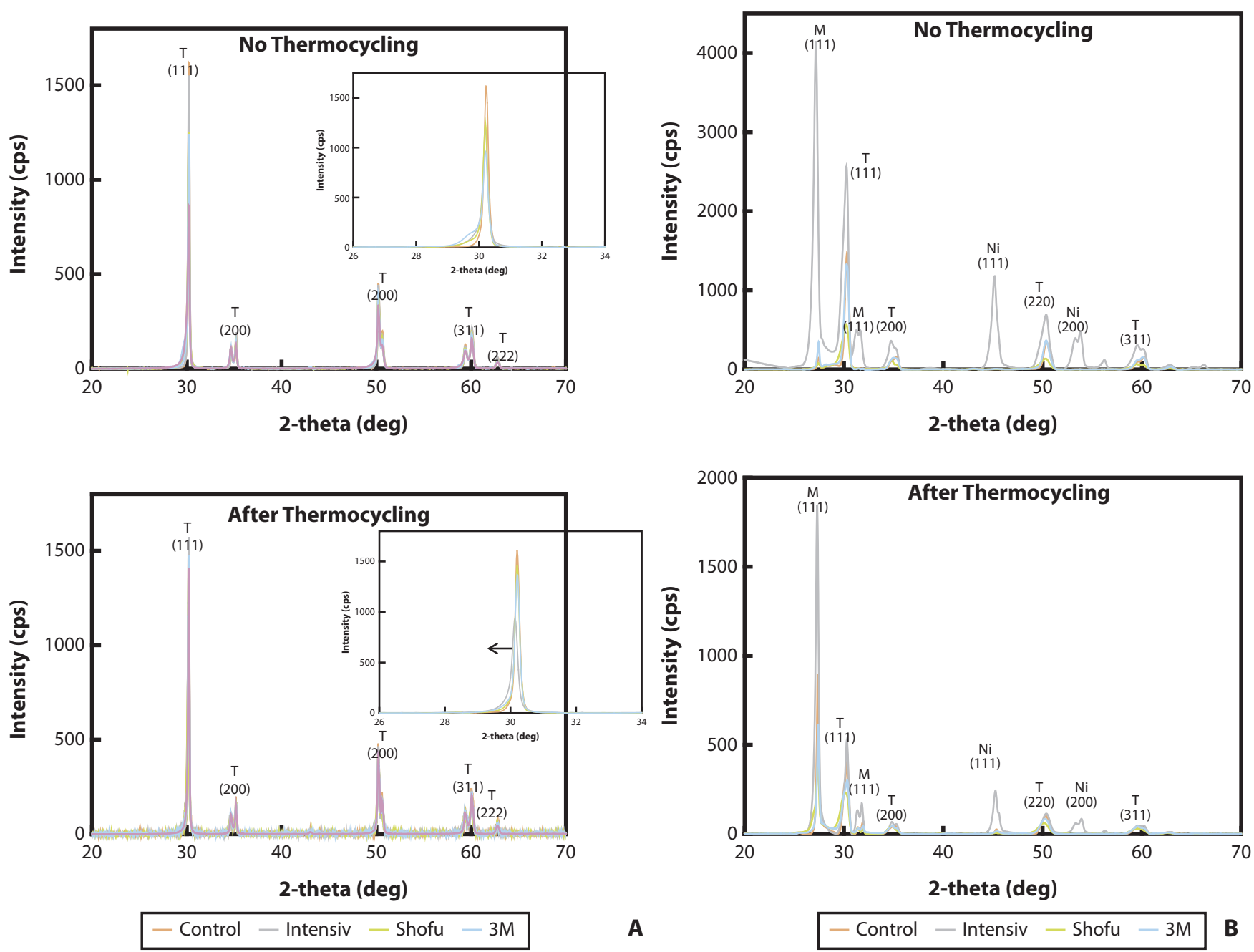

Figure 3. A, X-ray diffraction analysis of zirconia showing main crystalline phase tetragonal yttrium zirconium oxide (International Centre for Diffraction Data [ICDD]: 04-016-2103) before and after thermocyling and peak shift in Intensiv polishing method after thermocycling (inset) (control, Intensiv, Shofu, 3M ESPE). B, X-ray diffraction analysis in grazing angle of zirconia showing both main crystalline phase tetragonal yttrium zirconium oxide (T; ICDD: 04-016-2103) with additional monoclinic phase (M; ICDD: 04-005-4476) and traces of nickel (ICDD: 04-013-4763) in Intensiv polishing method before and after thermocyling (control, Intensiv, Shofu, 3M ESPE).
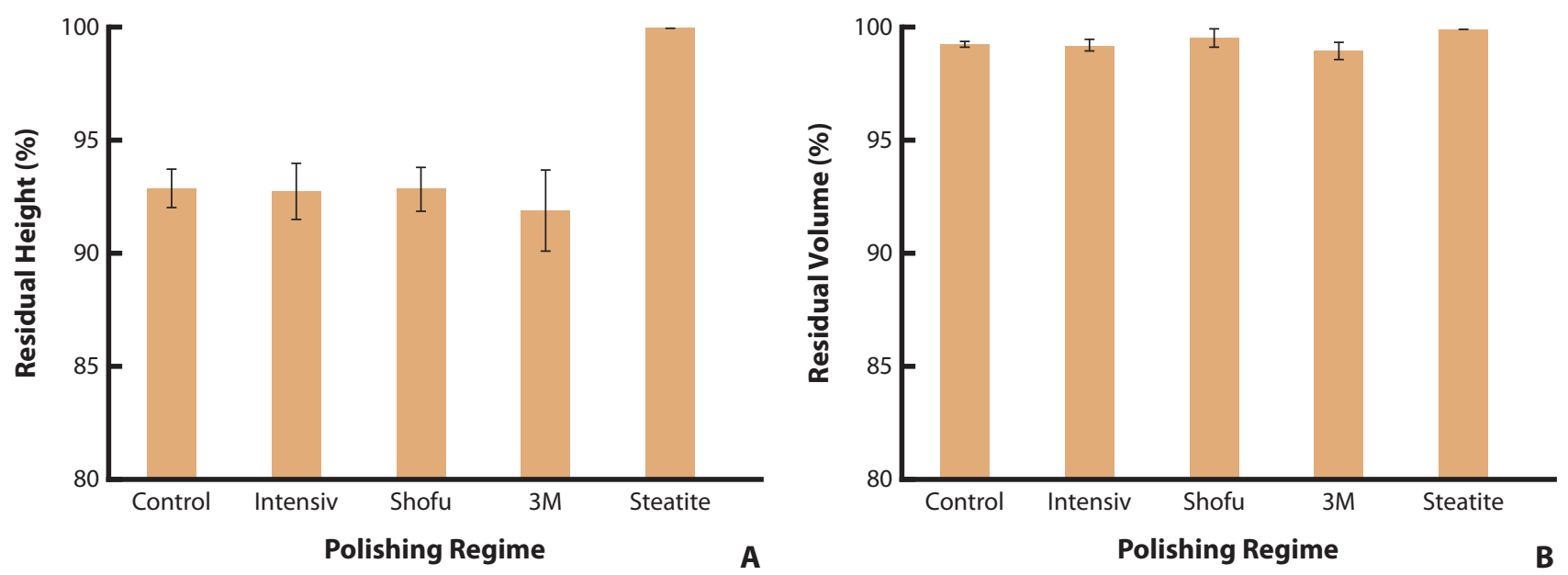

Figure 4. Height and volume changes of zirconia and polished zirconia in contact with steatite antagonist during mastication simulation. 
Specific conditions were used for mastication simulation and aging to simulate clinical condtions. ${ }^{21-25}$ The experiment was performed underwater, which served as a medium for the removal of any debris from the antagonist and partially simulated the oral environment. The use of steatite balls simulated human enamel when occluded with zirconia in the mastication simulator. Wear by the zirconia specimens was analyzed after the predetermined amount of cycles in the mastication simulator, which indicated the process of wear through time. The use of distilled water as a medium instead of saliva and the use of steatite balls rather than human enamel are limitations of this study.

Results from the mastication simulator have shown that the steatite balls in contact with zirconia were worn compared with the unworn steatite ball, but the volume lost was not significant. The polished zirconia and the control did not differ significantly in wear height and wear volume. Yttria-stabilized zirconia wears enamel with time, as shown in a recent study, ${ }^{26}$ but the surface roughness produced by polishing the specimens did not generate significant wear on the antagonists.

Aging was shown to reduce the surface roughness of yttria-stabilized zirconia, and the resultant wear of the antagonist tooth could reduce with time, unlike with other ceramic materials. This is in contrast to other reports on the effects of aged zirconia when in contact with enamel. ${ }^{27}$ After artificial aging of zirconia using autoclave and testing, no significant difference between aged and untreated zirconia was observed, as it affected enamel in the same way. ${ }^{27}$

\section{CONCLUSIONS}

On the basis of the findings of this in vitro study, the following conclusions were drawn:

1. Polishing monolithic zirconia increased surface roughness and led to surface phase changes.

2. Wear to the antagonist was not affected.

\section{REFERENCES}

1. Gupta TK, Lange FF, Bechtold JH. Effect of stress-induced phase transformation on the properties of polycrystalline zirconia containing metastable tetragonal phase. J Mater Sci 1978;13:1464-70.

2. Manicone PF, Rossi Iommetti P, Raffaelli L. An overview of zirconia ceramics: basic properties and clinical applications. J Dent 2014;35:819-26.

3. Garvie RC, Hannink RH, Pascoe RT. Ceramic steel? Nature 1975;258:703-4.

4. İseri U, Özkurt Z, Yalnız A, Kazazoğlu E. Comparison of different grinding procedures on the flexural strength of zirconia. J Prosth Dent 2012;107: 309-15.

5. Song JY, Park SW, Lee K, Yun KD, Lim HP. Fracture strength and micro structure of Y-TZP zirconia after different surface treatments. J Prosthet Dent 2014;110:274-80.
6. Tada K, Sato T, Yoshinari M. Influence of surface treatment on bond strength of veneering ceramics fused to zirconia. Dent Mater J 2012;31:287-96.

7. Kosmač T, Oblak C, Jevnikar P, Funduk N, Marion L. The effect of surface grinding and sandblasting on flexural strength and reliability of Y-TZP zirconia ceramic. Dent Mater 1999;15:426-33.

8. Guazzato M, Quach L, Albakry M, Swain MV. Influence of surface and heat treatments on the flexural strength of Y-TZP dental ceramic. J Dent 2005;33: 9-18.

9. Hallmann L, Ulmer P, Wille S, Polonskyi O, Köbel S, Trottenberg T, et al Effect of surface treatments on the properties and morphological change of dental zirconia. J Prosthet Dent 2016;115:341-9.

10. Traini T, Gherlone E, Parabita S, Caputi S, Piattelli A. Fracture toughness and hardness of a Y-TZP dental ceramic after mechanical surface treatments. Clin Oral Investig 2014;18:707-14.

11. Yener E, Özcan M, Kazazoğlu E. The effect of glazing on the biaxial flexural strength of different zirconia core materials. Acta Odontol Latinoam 2011;24: 133-40.

12. Xie H, Gu Y, Li Q, Qian M, Zhang F, Tay FR, Chen C. Effects of multiple firings on the low-temperature degradation of dental yttria-stabilized tetragonal zirconia. J Prosthet Dent 2016;115:495-500.

13. Huh YH, Park CJ, Cho LR. Evaluation of various polishing systems and the phase transformation of monolithic zirconia. J Prosthet Dent 2016;116:440-9.

14. Basílio Mde A, Cardoso KV, Antonio SG, Rizkalla AS, Santos Junior GC, et al. Effects of artificial aging conditions on yttria-stabilized zirconia implant abutments. J Prosthet Dent 2016;116:277-85.

15. Kern M, Wegner SM. Bonding to zirconia ceramic: adhesion methods and their durability. Dent Mater 1998;14:64-71.

16. Stawarczyk B, Özcan M, Schmutz F, Trottmann A, Roos M, Hämmerle CHF Two-body wear of monolithic, veneered and glazed zirconia and their corresponding enamel antagonists. Acta Odontol Scand 2012;71:102-12.

17. Preis V, Weiser F, Handel G, Rosentritt M. Wear performance of monolithic dental ceramics with different surface treatments. Quintessence Int 2013;44: 393-405.

18. Preis V, Behr M, Handel G, Schneider-Feyrer S, Hahnel S, Rosentritt M. Wear performance of dental ceramics after grinding and polishing treatments. J Mech Behav Biomed Mater 2012;10:13-22.

19. Janyavula S, Lawson N, Cakir D, Beck P, Ramp LC, Burgess JO. The wear of polished and glazed zirconia against enamel. J Prosthet Dent 2014;109:22-9.

20. Kontos L, Schille C, Schweizer E, Geis-Gerstorfer J. Influence of surface treatment on the wear of solid zirconia. Acta Odontol Scand 2013;71:482-7.

21. Passos SP, Torrealba Y, Major P, Linke B, Flores-Mir C, Nychka JA. In vitro wear behavior of zirconia opposing enamel: a systematic review. J Prosthodont 2014;23:593-601

22. Sakaguchi RL, Douglas WH, DeLong R, Pintado MR. The wear of a posterior composite in an artificial mouth: a clinical correlation. Dent Mater 1986;2: 235-40.

23. Heintze SD. How to qualify and validate wear simulation devices and methods. Dent Mater 2006:22:712-34.

24. Gibbs CH, Mahan PE, Lundeen HC, Brehnan K, Walsh EK, Holbrook WB. Occlusal forces during chewing and swallowing as measured by sound transmission. J Prosthet Dent 1981;46:443-9.

25. Rues S, Huber G, Rammelsberg P, Stober T. Effect of impact velocity and specimen stiffness on contact forces in a weight-controlled chewing simulator. Dent Mater 2011;27:1267-72.

26. Caruana K, White AJ, Bubb N, Brunton PA. Abrasivity of yttria tetragonal zirconia polycrystal (Y-TZP) to dental enamel in comparison to noble white high palladium alloy. Int J Dent Sci Res 2015;2:2-7.

27. Burgess JO, Janyavula S, Lawson NC, Lucas TJ, Cakir D. Enamel wear opposing polished and aged zirconia. Oper Dent 2013;39:189-94.

Corresponding author:

Prof J. Camilleri

University of Malta

Department of Restorative Dentistry

Faculty of Dental Surgery

MALTA

Email: josette.camilleri@um.edu.mt

\section{Acknowledgments}

The authors thank Ing James Camilleri, Biomaterials Research Fund, Department of Metallurgy and Materials Engineering, Faculty of Engineering, University of Malta and Tom Bull, Taicaan Technologies, for help with the analysis.

Copyright @ 2016 by the Editorial Council for The Journal of Prosthetic Dentistry. 